\title{
Active Expression of Retroelements in Neurons Differentiated from Adult Hippocampal Neural Stem Cells
}

\author{
Slawomir Antoszczyk, Kazuyuki Terashima, Masaki Warashina, \\ Makoto Asashima and Tomoko Kuwabara \\ Research Center for Stem Cell Engineering, \\ National Institute of Advanced Industrial Science and Technology (AIST) \\ Japan
}

\section{Introduction}

In the mammalian brain, neurogenesis constitutively occurs in the subventricular zone (SVZ), the olfactory bulb, and the hippocampus throughout adulthood (Kuhn et al., 1996; Lois and Alvarez-Buylla, 1993; Gage, 2000; Pagano et al., 2000; Gritti et al., 2002), and adult hippocampal neurogenesis plays an important role in learning and memory (Deng et al., 2010). In the hippocampus, multipotent neural stem cells (NSCs) reside in the inner layer of the subgranular zone (SGZ) of the dentate gyrus (Gage, 2000; Suh et al., 2007). Undifferentiated NSCs express the high mobility group (HMG)-box transcription factor Sox2 (D'Amour and Gage, 2003; Suh et al., 2007). Sox2 is an SRY-related transcription factor encoding an HMG DNA-binding motif, and is expressed in embryonic stem (ES) cells and neural epithelial cells during development (Avilion et al., 2003; Ferri et al., 2004). Sox2 is essential for the multipotency and self-renewal capacity of NSCs and also functions in pluripotent ES cells (Bylund et al., 2003; Graham et al., 2003; Ferri et al., 2004). Sox2 is known to prevent neurogenesis during development and is thought to be critical for maintaining NSC populations in the neonatal brain (Bylund et al., 2003; Graham et al., 2003; Bani-Yaghoub et al., 2006). In the dentate gyrus of the hippocampus, Sox2 expression is found in the undifferentiated stem cell population with self-renewal capacity, and these Sox2-positive stem cells are exclusive with TUJ1-positive early stage of neurons (Fig. 1).

Fate-tracing studies showed that Sox2-positive cells in the SGZ of the adult hippocampus have the potential to give rise to neurons and astrocytes, revealing their multipotency at both the cell population and single cell levels (Suh et al., 2007). Moreover, a subpopulation of Sox2-positive cells gives rise to cells that retain Sox2, highlighting the importance of Sox2positive cells as a primary source of adult NSCs (Suh et al., 2007). In response to intracellular or extracellular signals, Sox2-positive NSCs undergo cell division, giving rise to more Sox2positive NSCs as well as neuronal precursors (Avilion et al., 2003; Ferri et al., 2004). Adult hippocampal neural stem cells express receptors and signaling components for Wnt proteins, which are key regulators of NSCs (Lie et al., 2005). 

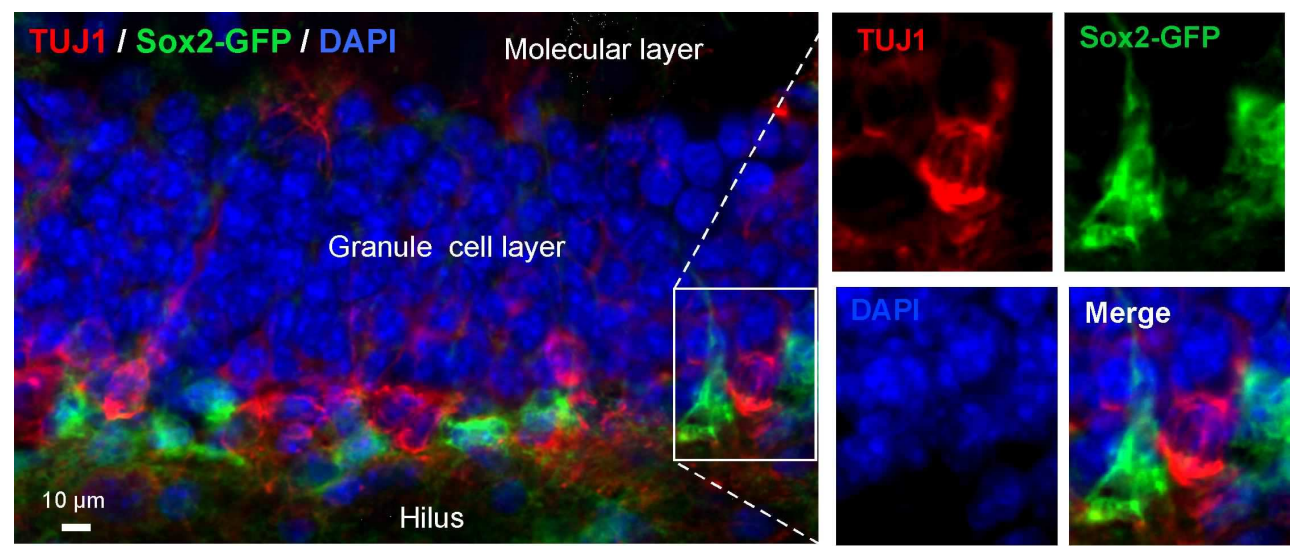

Hippocampal neural stem cells express multipotent marker Sox2. Sox2-positive cells (Sox2-GFP: green; immunohistochemistry analysis using Sox2 promoter-driven EGFP transgenic mice) are exclusive with early stage of neurons expressing $\beta$ tubulin III (TUJ1: red). DAPI; blue.

Fig. 1. Adult neural stem cells in dentate gyrus of hippocampus

\section{Wnt3 promotes the active expression of retroelements in adult hippocampal neurogenesis}

Astrocytes are an essential cell population defining the hippocampal niche (Song et al., 2002), and Wnt3 factors secreted from these cells are instructive in promoting adult neurogenesis (Lie et al., 2005). The deletion of Wnt3a (Wnt3a-/- mice) prevents the formation of the dentate gyrus, which is the site of adult neurogenesis (Lee et al., 2000). Other Wnt proteins (Wnt1, Wnt2, Wnt5a, and Wnt7a/b) are detected and actively function in mature hippocampal neurons (Miyaoka T et al., 1999; Gogolla et al., 2009; Cuitino et al., 2010; Okamoto et al., 2010), suggesting that other Wnt proteins work in an autocrine manner to control neuronal functions, activities in the neuronal network, and synaptic connectivity in mature neurons. In contrast, astrocyte-secreted Wnt3/Wnt3a (Lie et al., 2005) acts in a paracrine manner to induce neurogenesis in NSCs by direct activation of the proneuronal gene neurogenic differentiation 1 (NeuroD1), and to generate diversity in newborn neurons through retroelements (i.e., retrotransposons) (Kuwabara et al., 2009).

\subsection{Astrocytes-secreting Wnt3 initiates the expression of retroelements in adult neural stem cells}

We recently reported that Wnt3/Wnt3a released from underlying astrocytic layers in the dentate gyrus has an important role in triggering neuronal differentiation of hippocampal NSCs. NeuroD1, a target gene of Wnt signaling in the coding region of the mammalian genome, is a proneural basic helix-loop-helix (bHLH) transcription factor that is essential for the development of the CNS, particularly for the generation of granule cells in the hippocampus (Miyata et al., 1999; Liu et al., 2000; Deisseroth et al., 2004; Tozuka et al., 2005). The deletion of $\beta$-catenin leads to substantial loss of NeuroD1-positive cells, while the stem cell compartment remains intact in vivo, suggesting that $\mathrm{Wnt} / \beta$-catenin-mediated neuronal differentiation is dependent on NeuroD1 (Kuwabara et al., 2009). 
Importantly, paracrine Wnt factors secreted from astrocytes simultaneously target the genomic noncoding region through long interspersed nuclear elements (LINE-1, L1) (Kuwabara et al., 2009). L1 is a large family of mobile elements that constitutes up to $17 \%$ of the mammalian genome (Han and Boeke, 2005) and was recently found to be actively retrotransposed in the course of adult neurogenesis in rodents (Muotri et al., 2005) and humans (Coufal et al., 2009). The regulatory sequence recognized by both Sox 2 and $\mathrm{TCF} / \mathrm{LEF} / \beta$-catenin is present in the promoter region of NeuroD1 and L1 (Kuwabara et al., 2009). Sox 2 can suppress L1 expression in adult NSCs. In contrast, canonical Wnt/ $\beta$-catenin signaling triggers the active expression of NeuroD1 and L1, indicating the essential role of Sox2 and Wnt in balancing self-renewal of NSCs and neuronal differentiation in the adult hippocampal dentate gyrus (Kuwabara et al., 2009).

The balance of asymmetric lineage control of adult NSCs in maintaining the constant size of the neural stem cell pools and producing newly born neurons relies on the definitive molecular mechanism of Wnt target genes: the Sox/LEF overlapping regulatory sequence on NeuroD1 and L1 functions as a "molecular switch" between Sox2-mediated repression and Wnt signaling-mediated activation of target genes (Fig. 2).

\section{Neural stem cell}

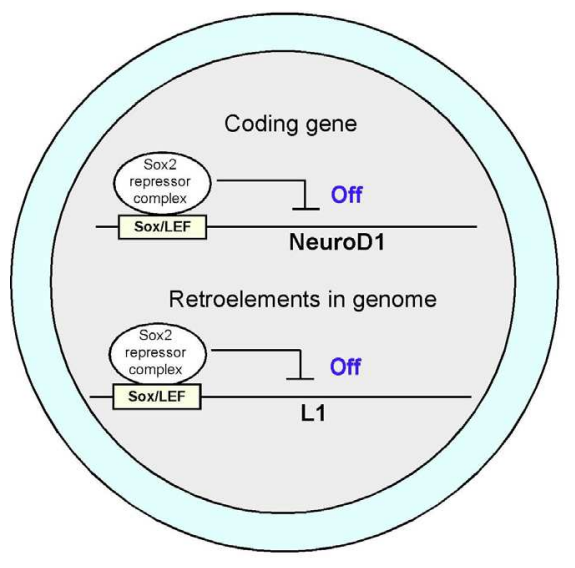

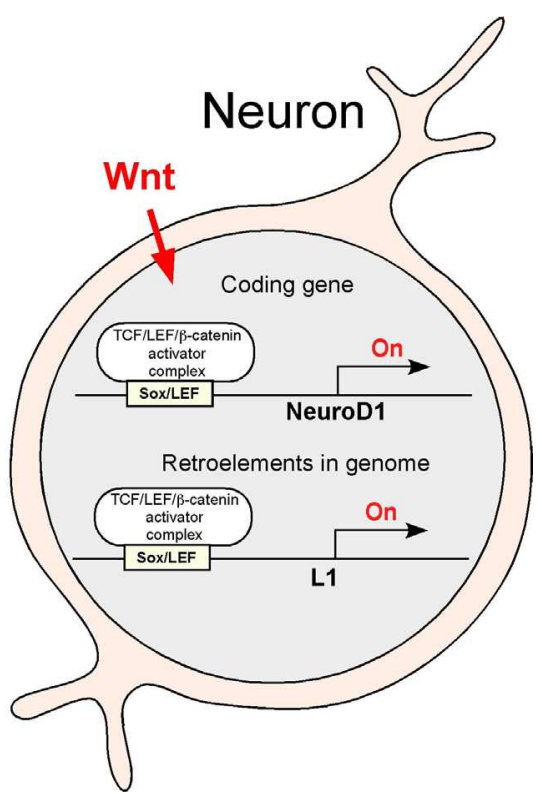

Overlapping Sox/LEF binding site is shown in yellow box. The overlapping DNA regulatory consensus sequence (A/T-A/T-C-A-A-A-G; yellow box) recognized by both Sox2 (A/T-A/T-C-A-A-A/T-G) and TCF/LEF (A/T-A/T-C-A-A-A). When Wnt3/Wnt3a stimulate NSCs, the Wnt-signaling activate NeuroD1 gene and L1 gene.

Fig. 2. Schematic representation of Wnt-mediated regulation in adult hippocampal NSCs.

The Sox/LEF regulatory elements reside within the 5' UTR sequences of human, rat, and mouse L1. Several Sox/LEF-binding sites are also present throughout the entire L1 sequence, including several sites in the second open reading frame (ORF2). The discovery 
that L1 retroelements embedded in the mammalian genome can function as bidirectional promoters suggests that Sox/LEF regulatory sites may represent a general mechanism for transcriptional regulation. This led us to examine whether other retroelements have a similar ability, thereby expanding the role of retroelements in adult hippocampal neurogenesis.

\subsection{Expression of B1 SINE RNA and B2 SINE RNAs in adult hippocampus}

The human and rodent genomes harbor numerous non-autonomous retrotransposons, termed short interspersed elements (SINEs). SINEs are highly abundant components of mammalian genomes and are propagated via retrotransposition (Ferrigno et al., 2001). Nonautonomous SINEs recruit L1-encoded proteins for their own mobilization. Alu elements are the major SINEs in the human genome, whereas B1 and B2 elements are the major SINE families in the mouse genome. The B2 SINE family constitutes approximately $0.7 \%$ of total mouse genomic DNA (Bennett et al., 1984). These retroelements are widely distributed throughout the genome, although many are heavily truncated and only a few are thought to be active and able to retrotranspose.

We first investigated B2 SINE RNA expression in the adult hippocampus by in situ hybridization. Brains were dissected from freshly euthanized Fisher 344 rats and placed in ice-cold saline. The brains were then placed in plastic blocks in OCT compound (Tissue Tek) and frozen. Sections were cut at $15 \mu \mathrm{m}$ thickness with a cryostat (LEICA CM1850, Leica). Brain sections on the slide glass were hybridized with labeled riboprobes. Following the in situ hybridization of B2 SINE RNA, immunohistochemical analysis of the L1 protein (1:300; rabbit antibody against LINE-1, SantaCruz) was carried out. We observed that hippocampal granule neurons extensively express L1 RNA, as well as B2 SINE RNA (Fig. 3). Strong signals were also observed in neuronal layers (granule cell layers) but were not found in cells at the innermost layer of the dentate gyrus where astrocytes and undifferentiated neural stem cells reside (Fig. 3).

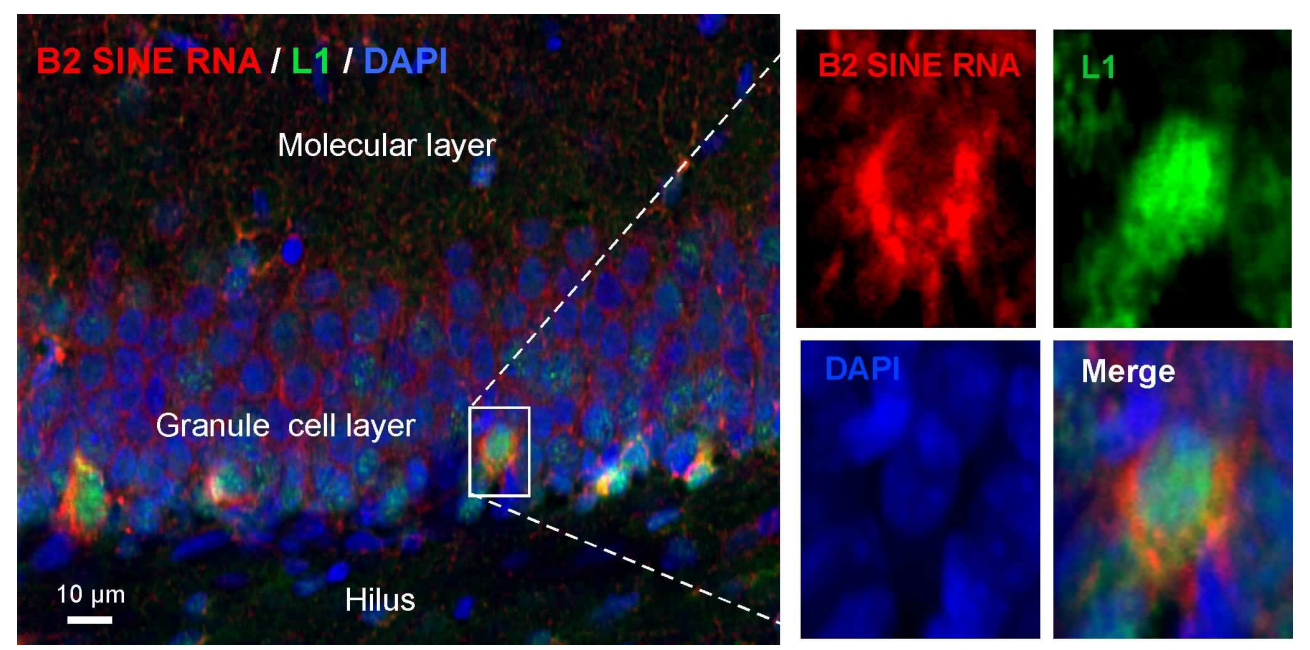

Fig. 3. Expression B2 SINE RNA and L1 in DG of adult hippocampus. 
In situ hybridization for B2 SINE RNA (red) and immunohistochemistry of L1 protein (green) was carried out simultaneously on the DG of adult rat hippocampus. Hippocampal granule neurons extensively express both B2 SINE RNA and L1. The SINE RNA and L1 protein double-positive cells in the white square are magnified in the right panels. B2 SINE RNA: red, L1 protein: green, DAPI: blue.

\subsection{Wnt-signaling regulatory sites on B1 SINE and B2 SINE DNA sequences}

We identified that the 2 major classes of non-coding retroelements, B1 and B2 SINEs (B1 SINE, GenBank accession number X62249; B2 SINE, M31441) also carry Wnt-responsive elements (Fig. 4). SINEs originate from retrotransposition events of small RNAs (Batzer and Deininger, 2002; Hasler \& Strub, 2006; Nishihara et al., 2006). Both Alu and B1 elements are derived from the 7SL RNA (Ullu \& Tschudi; 1984), whereas B2 and most other SINEs are derived from tRNA genes. The eukaryotic RNA polymerase III (Pol III) system is responsible for synthesizing transfer RNA molecules and other transcripts, which in yeast include the U6 spliceosomal RNA, 7SL RNA, 5S ribosomal RNA, snr52 small nucleolar RNA, and the RNA component of RNaseP (Paule \& White, 2000; Geiduschek \& Kassavetis, 2001; Huang \& Maraia, 2001). Transcription Factor for polymerase III C (TFIIIC) binds to 2 intragenic (lying within the transcribed DNA sequence) control sequences, the A-Box and B-Box (Fig. 4).

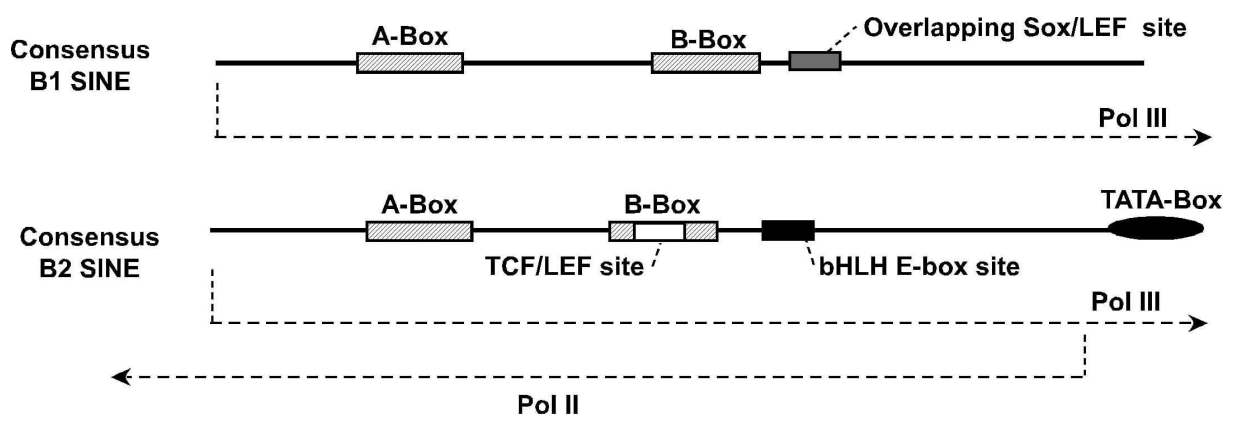

Fig. 4. Transcriptional regulation of B1 SINE RNA and B2 SINE RNA.

RNA polymerase III (also called Pol III) transcribes DNA by recognizing A-box and B-box (grey boxes). Overlapping Sox/LEF sequence (dark grey box) is contained in B1 SINE RNA. TCF/LEF regulatory sequence (white box) is involved in the B-box sequence of consensus B2 SINE RNA. The DNA of B2 SINE RNA contains also the bHLH transcription factor recognizing E-box sequence (black box). B2 SINE also carries an active RNA polymerase II (pol II) regulatory site that is located outside the tRNA region (Ferrigno et al., 2001). TATA box of the pol II promoter is indicated in black oval box.

As Figure 4 indicates, DNA sequences of both B1 SINE RNA and B2 SINE RNA include a Wnt signaling responsive element (Sox/LEF site in B1 SINE and TCF/LEF site in B2 SINE). Interestingly, the DNA sequence of B2 SINE RNA also contains an E-box sequence, which is recognized by a bHLH transcription factor, such as NeuroD1 (Fig. 4). The expression of NeuroD1 is triggered by Wnt-signaling, suggesting that B2 SINE RNA has additional active sites for NeuroD1 regulation in the adult neuronal lineage-differentiation. To explore the expression profile of these retroelement RNAs (B1 SINE RNA and B2 SINE RNA) in a lineage-specific manner in the adult hippocampus, we cultured adult hippocampal NSCs. 


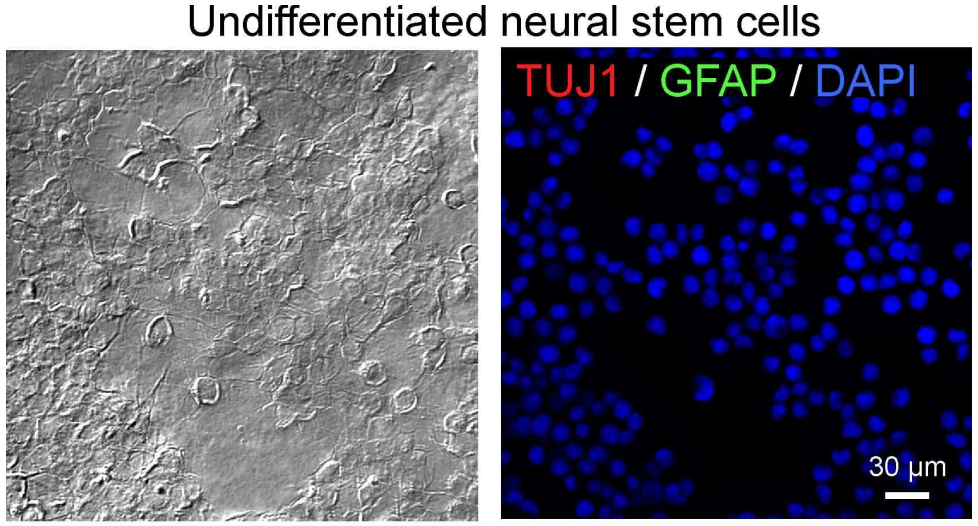

\section{Neurons}
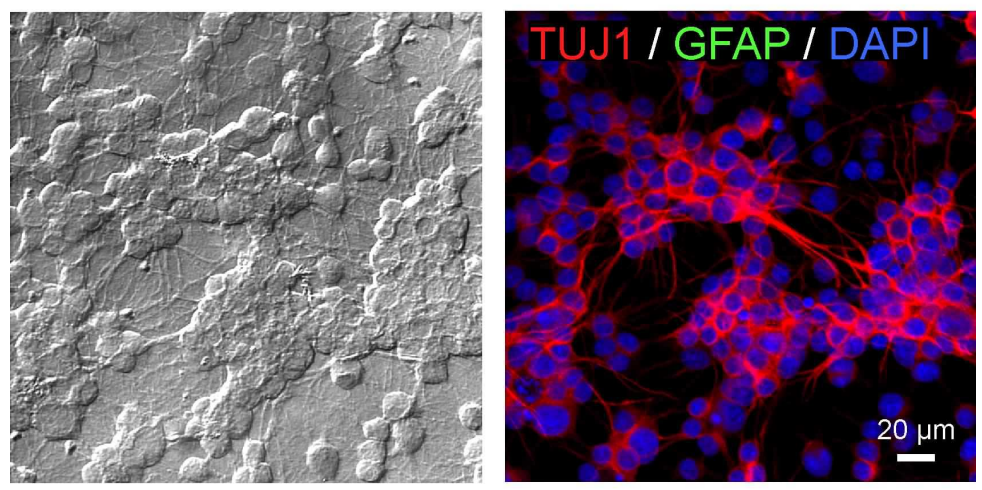

\section{Astrocytes}
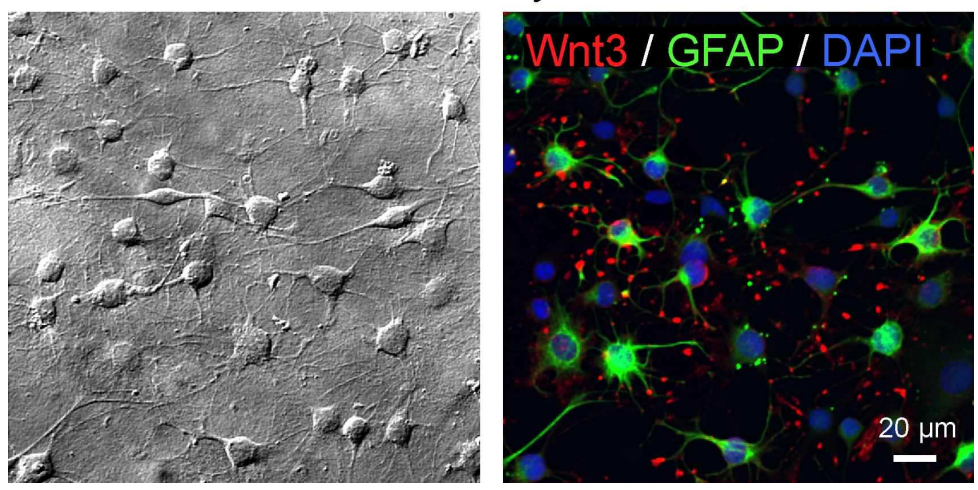

Neural stem cells (top), neurons (middle) and astrocyte cells (bottom) were examined by the immunohistochemistry analysis. TUJ1 (top and middle) and Wnt3 (bottom): red, GFAP: green, DAPI: blue.

Fig. 5. In vitro culture system of adult hippocampal neural stem cells 


\subsection{Expression of B1 SINE RNA and B2 SINE RNA in adult hippocampal neural stem cell culture}

Adult hippocampal NSCs were round and retained their shape when expanded as a monolayer (top panels, Fig. 5). These neural progenitor cells have stem cell properties in vitro: (1) they undergo self-renewal in the presence of basic FGF-2; (2) single genetically marked clones can differentiate into neurons, oligodendrocytes, and astrocytes in vitro and when grafted back to the adult hippocampus in vivo; and (3) they express progenitor cell markers such as Sox2 and nestin (Gage et al., 1995; Palmer et al., 1997). Undifferentiated NSCs were negative for the neuronal marker $\beta$-tubulin III (TUJ1) and the astrocyte lineage marker glial fibrillary acidic protein (GFAP) (Fig. 5). Under neuronal differentiation conditions, we added $1 \mu \mathrm{M}$ retinoic acid and $5 \mu \mathrm{M}$ forskolin to the culture. The expression of $\beta$-tubulin III was remarkably up-regulated, as confirmed by the immunohistochemistry analysis. GFAP was found to be absent in the immunohistochemistry analysis, indicating that the adult NSCs in culture were committed to a neuronal lineage specifically (middle panels, Fig. 5). Differentiation into the astrocyte lineage was stimulated by the addition of $50 \mathrm{ng} / \mathrm{mL}$ leukemia inhibitory factor (LIF) and $50 \mathrm{ng} / \mathrm{mL}$ bone morphogenetic protein 2 (BMP2). Astrocytes prepared in vitro expressed Wnt3 factors, consistent with in vivo data (Lie et al., 2005; Kuwabara et al., 2009; Okamoto et al, 2011).

\subsection{Neuronal-specific expression of B1 SINE RNA and B2 SINE RNA in the adult hippocampal neural stem cell culture}

By using an in vitro culture system of adult hippocampal NSCs (Fig. 5), we investigated the relative expression levels of B1 SINE and B2 SINE RNA. Neurons differentiated from adult neural stem cells expressed high levels of both B1 RNA and B2 RNA, although the expression level of B2 SINE RNA was higher (Fig. 6).

Total RNAs were extracted cells and expression levels of B1 SINE RNA (black bars) and B2 SINE RNA (white bars) ware examined by quantitative real-time PCR (Q-PCR analysis). A) The level was normalized internal control gene GAPDH and platted in the graph. The expression level of B1 SINE RNA in undifferentiated neural stem cells (NSCs) was taken as 100\% (asterisk; black bars). B) Time course analysis of B1 SINE RNAs and B2 SINE RNAs.

In the astrocyte lineage, the basal expression of B1 SINE RNA and B2 SINE RNA from undifferentiated cells was largely diminished (Fig. 6A). The neuronal specific expression of B2 SINE RNA by quantitative real-time PCR (QPCR) analysis in vitro was consistent with the results of in situ hybridization (Fig. 3).

Time course of B1 SINE RNA and B2 SINE RNA expression during the early stages of neurogenesis in cultured adult NSCs was assessed by QPCR analysis. Following neuronal differentiation in vitro, B1 SINE RNA and B2 SINE RNA expression peaked at $24 \mathrm{~h}$ after neuronal induction (20-fold increase in B1 SINE RNA, 50-fold increase in B2 SINE RNA). These expression levels gradually decreased until they reached a plateau by $48 \mathrm{~h}$ after neuronal induction (Fig. 6B). Both NeuroD1 and L1 expression was highest at $24 \mathrm{~h}$ after neuronal induction and gradually declined during neuronal differentiation (Kuwabara et al., 2009), similar to the expression profile of B1 SINE RNA and B2 SINE RNA, suggesting 
that these genes are under similar transcriptional regulation. Combined expression analysis of in situ hybridization and immunohistochemistry of B2 SINE RNA and L1 showed that early stage neuronal progenitors (i.e., neuroblast cells) contained high levels of L1 and B2 SINE RNAs (Fig. 3; inner layer of dentate gyrus, cells indicated by the white square). In the mature neuronal layer of the dentate gyrus (deeper neuronal layer of SGZ), the L1 and B2 SINE RNA signals were still clearly observed, although it was weaker than those in neuroblast cells. The in vivo expression profile obtained from in situ hybridization and the in vitro expression profile obtained from the time course analysis using adult NSC culture are consistent (Fig. 3 and Fig. 6).

A

$\square$ B1 SINE $\square$ B2 SINE

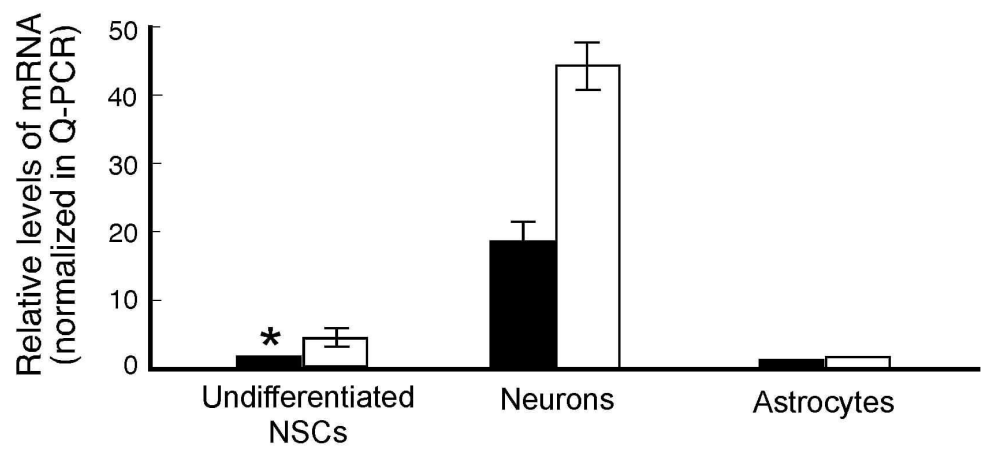

B

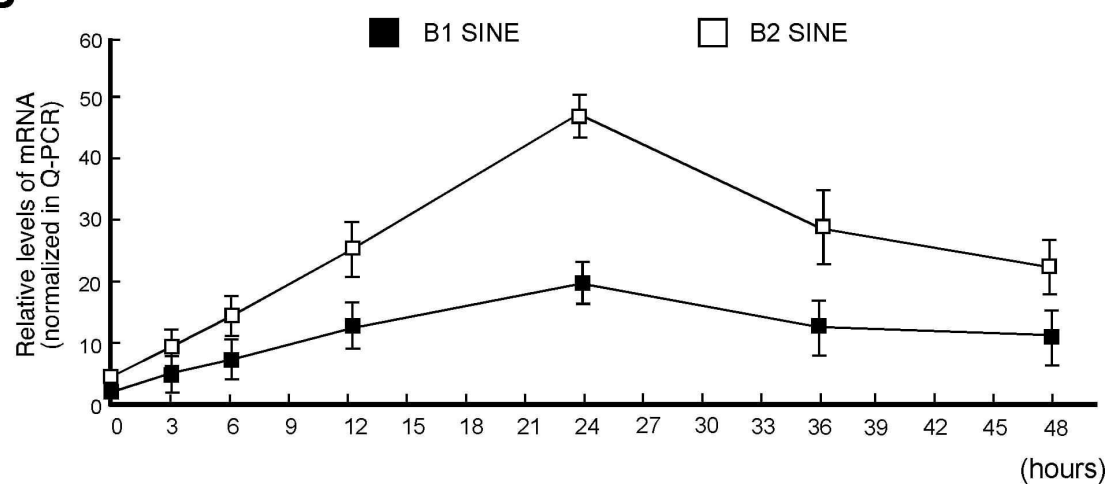

Fig. 6. Expression of B1 SINE RNA and B2 SINE RNA during adult neurogenesis.

\subsection{Effect of Wnt3 on the expression of B1 SINE RNA and B2 SINE RNA}

Next, we examined the effect of the Wnt3a ligand on the expression of B1 SINE RNA and B2 SINE RNA. Wnt3a ligands were added to the NSC culture at different concentrations (0 $\mathrm{ng} / \mathrm{mL}, 10 \mathrm{ng} / \mathrm{mL}, 20 \mathrm{ng} / \mathrm{mL}$, and $50 \mathrm{ng} / \mathrm{mL}$ of Wnt3a), and the expression levels of B1 SINE RNA and B2 SINE RNA were up-regulated in a dose-dependent manner (Fig. 7). 


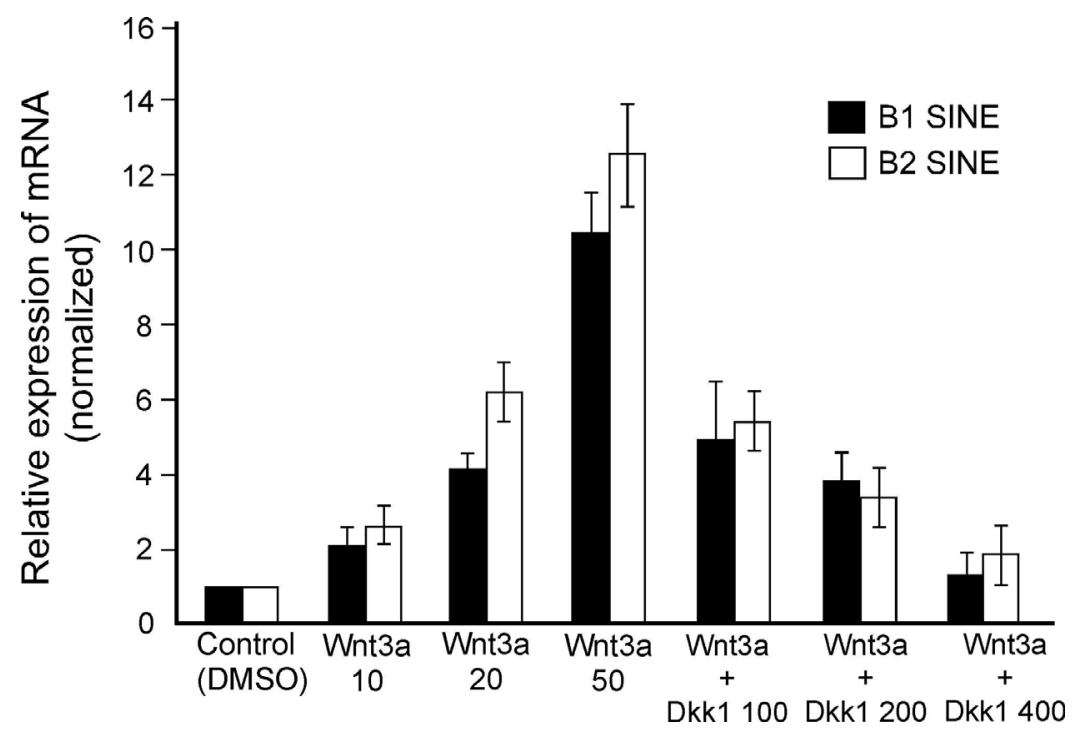

Fig. 7. Wnt signaling increases the expression of B1 SINE RNA and B2 SINE RNA in adult neural stem cell cultures. Ligand of Wnt3a and Dkk1 was added in the adult NSC cultures. Ligand concentration is indicated in the graph $(\mathrm{ng} / \mathrm{mL})$. The expression levels of B1 SINE RNA and B2 SINE RNA were normalized with internal control GAPDH. Cells treated with DMSO were taken as control and the relative value of the normalized expression of B1 SINE RNA and B2 SINE RNA was plotted in the graph. B1 SINE RNA: black bars, B2 SINE RNA: white bars.

To evaluate the observed positive effects of Wnt3a on the expression of B1 SINE RNA and B2 SINE RNA, the Wnt antagonist Dickkopf1 (Dkk1) was added into the adult hippocampal NSC culture. As the antagonist concentration was increased $(0 \mathrm{ng} / \mathrm{mL}, 100 \mathrm{ng} / \mathrm{mL}, 200$ $\mathrm{ng} / \mathrm{mL}$, and $400 \mathrm{ng} / \mathrm{mL}$ of Dkk1 with $50 \mathrm{ng} / \mathrm{mL}$ Wnt3a), Wnt3a-mediated activation of B1 SINE RNA and B2 SINE RNA was diminished (Fig. 7). From these data, we confirmed the contribution of Wnt signaling to the transcriptional activation of B1 SINE RNA and B2 SINE RNA in adult hippocampal NSCs.

\subsection{Effect of Wnt-signaling on the chromatin regulation of B1 SINE RNA and B2 SINE RNA}

To assess protein association on the regulatory region of B1 SINE RNA and B2 SINE RNA, we performed chromatin immunoprecipitation (ChIP). Association levels were quantitatively evaluated using real-time PCR (ChIP-QPCR). From in situ hybridization / immunohistochemistry in vivo data (Fig. 3) and the time course expression profile in vitro (Fig. 6), coordinated expression between SINE RNAs and L1 was observed. Therefore, we performed ChIP-QPCR analysis for SINE RNAs and L1 in parallel.

Addition of Wnt3a into the adult NSC culture promoted the enrichment of trimethyl histone H3 lysine 4 (triMetK4), a modified histone mark associated with gene activation, by more than 15-fold at the B1 SINE RNA locus and by more than 20-fold at the B2 SINE RNA locus, compared to the control cells treated with DMSO (Fig. 8). 

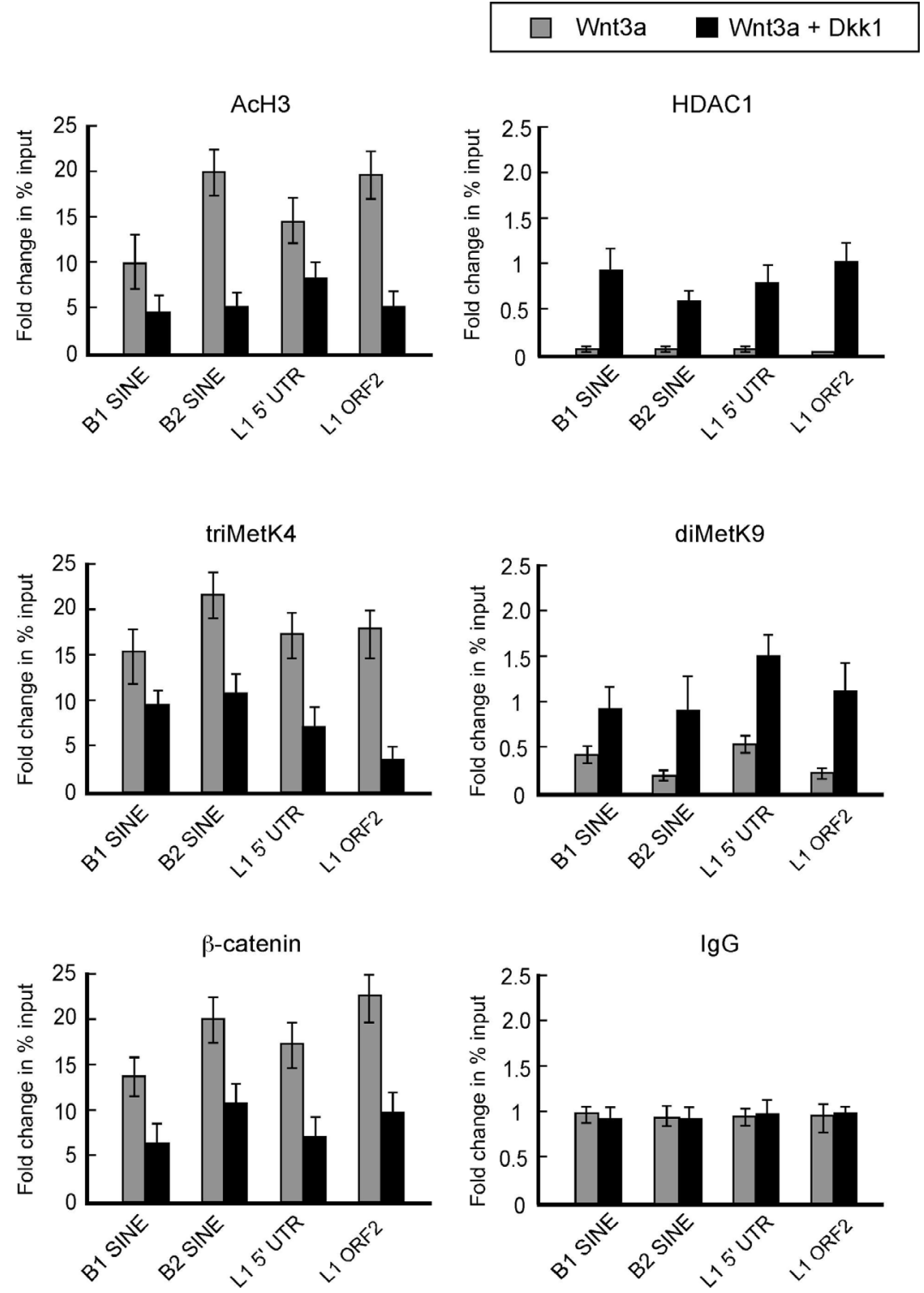

Fig. 8. Chromatin immunoprecipitation analysis (ChIP-QPCR) of retroelements in adult neural stem cells stimulated with Wnt3a and the antagonist Dkk1. PCR primers were designed to surround the Sox/LEF sequence on the L1 promoter. PCR primers for B1 SINE RNAs were designed to surround the Sox/LEF sequence on the B1 SINE DNA (Fig. 4). PCR primers for B2 SINE RNAs were designed to surround the TCF/LEF sequence and bHLH Ebox sequence on the B2 SINE DNA (Fig. 4). From these results, we confirmed that similar molecular mechanism controlled SINE RNAs and L1 in the adult hippocampal neurogenesis and Wnt3 has essential role to activate the transcription of these retroelements. 
The association of acetylated histone $\mathrm{H} 3$ (Ac-H3) and $\beta$-catenin increased with the Wnt3a treatment. Dimethylated H3 lysine 9 (diMetK9) and histone deacetylase 1 (HDAC1) were rarely associated, suggesting that the Wnt3a treatment promotes an active chromatin state (Fig. 6A). In contrast, the addition of Dkk1 inhibited the Wnt3a-mediated activation of chromatin at the B1 SINE RNA and B2 SINE RNA loci. We found that $\beta$-catenin, Ac-H3, and triMetK4 were associated at the L1 5' UTR region and L1 ORF2 region, both of which include the Sox/LEF regulatory sequence (Kuwabara et al., 2009), similar to their association on the B1 SINE RNA and B2 SINE RNA loci. In contrast, Dkk1 diminished the Wnt3a-mediated activation process, as seen in the case of the B1 SINE RNA and B2 SINE RNA loci (Fig. 8).

\subsection{Promoter activity of B2 SINE RNA in vivo}

Although we determined the global expression of B2 SINE RNA in neuronal cells by in situ hybridization (Fig. 3), we further examined the detailed expression of B2 SINE RNA in the adult hippocampus. The transcriptional activity of B2 SINE RNA is controlled by regulatory sequences present on the internal promoter (Fig. 4). We prepared a lentivirus construct that carries the B2 SINE promoter sequence (not including termination sequences) and EGFP reporter cassette (B2 SINE promoter driven EGFP; LV SINE GFP).

SINE GFP lentivirus was stereotactically injected into the dentate gyrus of young adult rats. Three weeks later, mice were injected with BrdU daily for 10 days. Notably, we observed that SINE GFP expression was restricted to neurogenic areas, and that GFP-positive cells colocalized with NeuroD1-positive neuronal progenitors (Fig. 9). We also detected SINE GFP-positive cells that migrated further into the granule cell layer where NeuN-positive mature neurons reside.
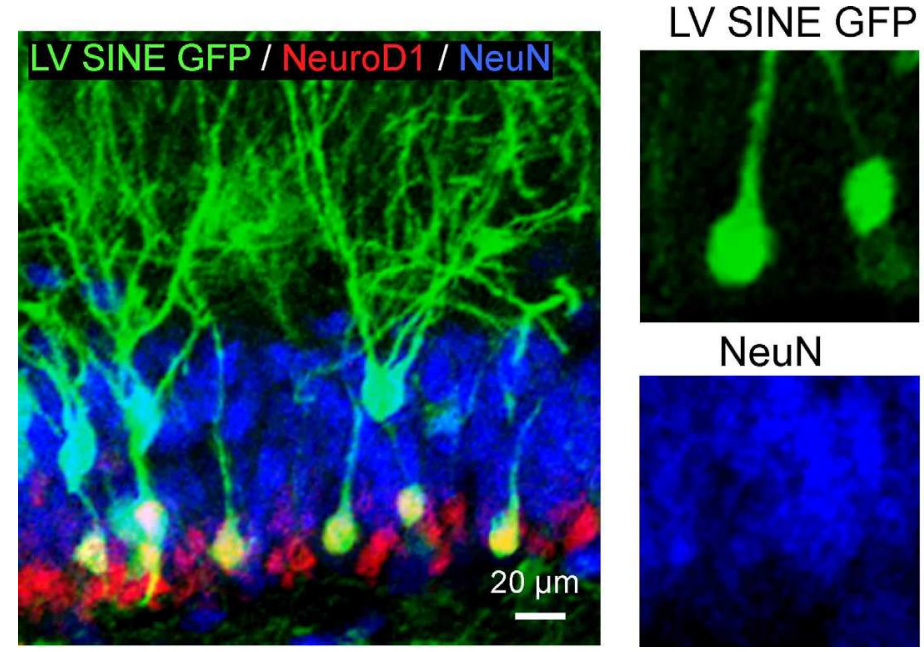

Fig. 9. Activity of B2 SINE as a promoter in adult rat hippocampus.

We examined the activity and specificity of the B2 SINE-based promoter in adult rat hippocampus. EGFP-expressing lentivirus, under the control of the B2 SINE-based promoter, was stereotactically microinjected into the dentate gyrus of adult rats and the 
population of GFP-positive cells (green) was analyzed by immunohistochemistry using antibodies to NeuroD1 (red) and NeuN (blue).

To identify the composition of cell types in which the B2 SINE-based promoter activity was turned on, we quantified the results of the immunohistochemical analysis. The number of SINE GFP and the lineage marker double-positive cells was counted and graphically plotted (Fig. 10).

A

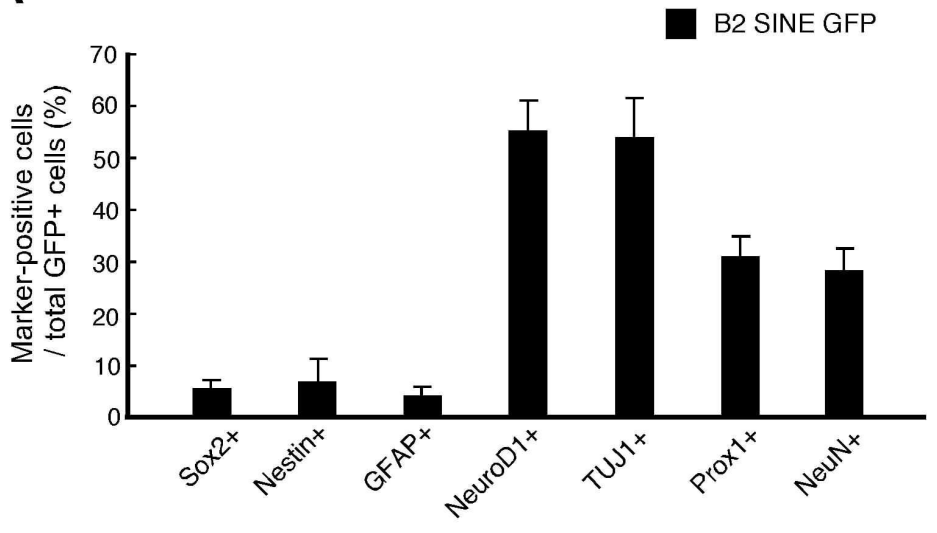

B

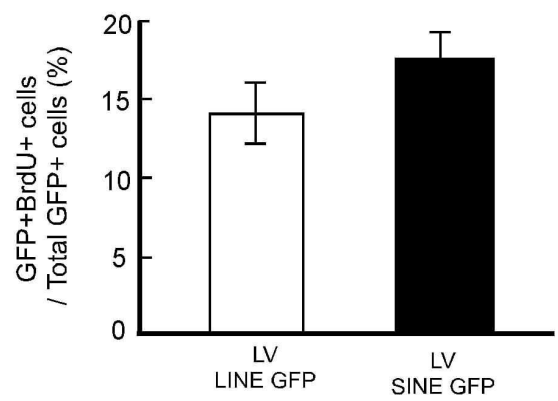

Fig. 10. Numbers of marker and SINE GFP double-positive cells in the dentate gyrus of adult rat. A) Quantitative immunohistochemistry analysis of B2 SINE promoter-active cells in dentate gyrus. B) Quantification of SINE GFP-positive and BrdU-positive cells in dentate gyrus of adult rat (black bar). LINE GFP-positive cells were also examined (white bar).

To examine SINE GFP-positive cells in the stem cell compartment, we stained the cells with Sox 2 and nestin, a marker of radial stem-like cells. The proportion of Sox2 and SINE GFP double-positive cells was $6.4 \%$ of the total SINE GFP-positive cells. The proportion of nestin and SINE GFP double-positive cells was $7.4 \%$ of the total SINE GFP-positive cells. To examine SINE GFP-positive cells in the glial cell compartment, we stained the cells with GFAP. The proportion of GFAP and SINE GFP double-positive cells was 3.7\% of total SINE GFP-positive cells. 
In contrast, SINE GFP-positive cells in the neuronal cell compartment comprised a majority of the total GFP-positive cells (Fig. 10A). B2 SINE-based promoter activity was up-regulated in cells at the early stage of neuronal lineage, such as NeuroD1- and TUJ1-positive cells. The proportion of NeuroD1 and SINE GFP double-positive cells was 56\% of the total SINE GFPpositive cells. The up-regulated activity of the SINE GFP promoter was retained in mature neurons (Prox-1- and NeuN-positive cells), but the proportion was almost half of neuroblast cells (NeuroD1-positive cells). Furthermore, SINE GFP-positive cells were labeled by BrdU, and the proportion of double-positive cells was found to be higher than that with LINE-GFP and BrdU staining (Fig. 10B), suggesting that the B2 SINE RNA-expressing cells actively proliferated in the adult hippocampus.

\section{Conclusion}

Our data indicate that both B1 SINE RNA and B2 SINE RNA are up-regulated during adult hippocampal neurogenesis, and that Wnt induction is required as the driving force. The specific response of these retroelements to paracrine Wnt factors is coupled with the differentiation progress of adult hippocampal NSCs into the neuronal pathway. Upon Wntmediated neuronal induction, the $\beta$-catenin activation complex up-regulated NeuroD1 and L1, as well as SINEs in adult hippocampal NSCs in vitro, while the Wnt antagonist Dkk1 down-regulated SINE RNA expression.

In ChIP-QPCR analysis, the association of $\beta$-catenin, triMetK4, and Ac-H3 in B1 and B2 SINEs was stimulated in cells treated with the Wnt3a ligand, suggesting that the genomic DNA of B1 and B2 SINEs was positively activated by canonical Wnt signaling. From these data, we propose that the activation of various genomic loci, where L1 and SINE fragments are present, may occur during adult hippocampal neurogenesis. Expression of retroelement RNAs are identified generally in neurogenic region in adult brain, although only hippocampal dentate gyrus is determined as the region that the retrotransposition (" jumping") occurs. Since various transcriptional activators are found in other neurogenic region in adult brain, not solely Wnt-signaling pathway but also other multiple molecules may control the expression of retroelement RNAs. We will actively extend current research to further determine potent regulators and the role of retroelement RNA during adult neurogenesis.

Since retroelement sequences are scattered throughout the genome and contain Wntresponsive regulatory elements, retroelements retain the ability to act as promoters inducing the activity of nearby chromatin loci during adult neurogenesis. The functional relevance of adult hippocampal neurogenesis has been extensively studied, and the fact that physiological and behavioral events, such as aging, stress, diseases, seizures, learning, and exercise, can modulate neurogenesis is of particular interest. This suggests that the chromatin state at SINE/L1 loci potentially changes depending on paracrine stimuli. These epigenetic mechanisms may act as genomic "sensors" of environmental changes and function as fine modulators of adult hippocampal neurogenesis.

\section{Acknowledgment}

We thank Hideto Takimoto for providing assistance in the care of animals and in the experiments. SA, MW, KT, MA and TK were supported by various grants from AIST. TK was partly supported by the Grant-in-Aid for Young Scientists (B). 


\section{References}

Avilion, . AA. Et al. (2003) Multipotent cell lineages in early mouse development depend on SOX2 function. Genes \& Development, Vol. 17, No. 1, (January 1), 126-140, ISSN 08909369

Bani-Yaghoub, M. et al. (2006) Role of Sox2 in the development of the mouse neocortex. Developmental Biology, Vol. 295, No. 1, (July 1), pp. 52-66, ISSN 0012-1606

Batzer, M. A. \& Deininger, P. L. (2002) Alu repeats and human genomic diversity. Nature Reviews Genetics, Vol. 3, No. 5, (May), pp. 370-379, ISSN 1471-0056

Bennett, K.L. et al. (1984) Most highly repeated dispersed DNA families in the mouse genome. Molecular and Cellular Biology. Vol. 4, No. 8, (August) 1561-1571, ISSN 0270-7306

Bylund, M. et al. (2003) Vertebrate neurogenesis is counteracted by Sox1-3 activity. Nature Neuroscience, Vol. 6, No. 11, (November), pp. 1162-1168, ISSN 1097-6256

Coufal, N. G. et al. (2009) L1 retrotransposition in human neural progenitor cells. Nature, Vol. 460, No. 7259, (August 27), pp. 1127-1131, ISSN 0028-0836

Cuitino, L. N. et al. (2010) Wnt5a is found to modulate recycling of functional GABAA receptors on hippocampal neurons. The Journal of Neuroscience, Vol. 30, No. 25, (June 23), pp. 8411-8420, ISSN 0270-6474

Deisseroth, K. et al. (2004) Excitation-neurogenesis coupling in adult neural stem/ progenitor cells. Neuron Vol. 42, No. 4, (May 27), pp. 535-552, ISSN 0896-6273

Deng, W. et al. (2010) New neurons and new memories: how does adult hippocampal neurogenesis affect learning and memory? Nature Reviews Neuroscience, Vol.11, No.5, (May 11), pp. 339-350, ISSN 1471-0048

Ferri, A. L. et al. (2004) Sox2 deficiency causes neurodegeneration and impaired neurogenesis in the adult mouse brain. Development, Vol. 131, No. 15, (August), pp. 3805-3819, ISSN 1011-6370

Ferrigno, O. et al. (2001) Transposable B2 SINE elements can provide mobile RNA polymerase II promoters. Nature Genetics, Vol. 28, No. 1, (May 28), pp. 77-81, ISSN 1061-4036

Gage, F. H. (2000) Mammalian neural stem cells. Science Vol.287, No.5457, (February 25), pp.1433-1438, ISSN 1095-9203.

Gage, F. H. et al. (1995) Survival and differentiation of adult neuronal progenitor cells transplanted to the adult brain. Proceedings of the National Academy of Sciences, Vol. 92, No.25, (December 5), pp. 11879-11883, ISSN 1091-6490

Geiduschek, E. P. \& Kassavetis, G. A. (2001) The RNA polymerase III transcription apparatus. Journal of Molecular Biology Vol. 310, No. 1, (June 29), pp. 1-26, ISSN 0022-2836

Gogolla, N. et al. (2009) Wnt signaling mediates experience-related regulation of synapse numbers and mossy fiber connectivities in the adult hippocampus. Neuron Vol. 62, No. 4, (May 28), pp. 510-525, ISSN 0896-6273

Graham, V. et al. (2003) SOX2 functions to maintain neural progenitor identity. Neuron, Vol. 39, No. 5, (August 28) pp. 749-765, ISSN 0896-6273

Gritti, A. et al. (2002) Multipotent neural stem cells reside into the rostral extension and olfactory bulb of adult rodents. The Journal of Neuroscience, Vol.22, No.2, (January 15), pp. 437-445, ISSN 0270-6474 
Han, J. S. \& Boeke, J. D. (2005) LINE-1 retrotransposons: modulators of quantity and quality of mammalian gene expression? Bioessays, Vo. 27, No. 8, (August), pp. 775-784, ISSN 1521-1878

Häsler, J. \& Strub, K. (2006) Alu elements as regulators of gene expression. Nucleic Acids Research, Vol. 34, No. 19, pp. 5491-5497, ISSN 0305-1048

Huang, Y. \& Maraia, RJ. (2001) Comparison of the RNA polymerase III transcription machinery in Schizosaccharomyces pombe, Saccharomyces cerevisiae and human. Nucleic Acids Research, Vol. 29, No. 13, (July 1), pp. 2675-2690, ISSN 0305-1048

Kuhn, H. G. et al. (1996) Neurogenesis in the dentate gyrus of the adult rat: age-related decrease of neuronal progenitor proliferation. The Journal of Neuroscience, Vol.16, No.6, (March 15), pp. 2027-2033, ISSN 0270-6474

Kuwabara, T. et al. (2009) Wnt-mediated activation of NeuroD1 and retro-elements during adult neurogenesis. Nature Neuroscience, Vol. 12, No. 9, (September), pp. 1097-1105, ISSN 1097-6256

Lee, S. M. et al. (2000) A local Wnt-3a signal is required for development of the mammalian hippocampus. Development, Vol. 127, No. 3, (February), pp. 457-467, ISSN 1011-6370

Lie, D. C. et al. (2005) Wnt signalling regulates adult hippocampal neurogenesis. Nature, Vol. 437, No. 7063, (October 27), pp. 1370-1375, ISSN 0028-0836

Liu, M. et al. (2000) Loss of BETA2/NeuroD leads to malformation of the dentate gyrus and epilepsy. Proceedings of the National Academy of Sciences, Vol.97, No2, (January 18), pp. 865-870, ISSN 1091-6490

Lois, \&Alvarez-Buylla, A. (1993) Proliferating subventricular zone cells in the adult mammalian forebrain can differentiate into neurons and glia. Proceedings of the National Academy of Sciences, Vol.90, No.5, (March 1), pp. 2074-2077, ISSN 1091-6490

Miyaoka, T., Seno, H., and Ishino, H. (1999) Increased expression of Wnt-1 in schizophrenic brains. Schizophrenia Research, Vol. 38, No. 1, (July 27), pp. 1-6, ISSN 0920-9964

Miyata, T. et al. (1999) NeuroD is required for differentiation of the granule cells in the cerebellum and hippocampus. Genes E Development, Vol. 13, No. 13, (Jury 1), 14671652, ISSN 0890-9369

Muotri, A. R. et al. (2005) Somatic mosaicism in neuronal precursor cells mediated by L1 retrotransposition. Nature, Vol. 435, No. 7044, (June 16), pp. 903-910, ISSN 00280836

Nishihara, H. et al. (2006) Functional noncoding sequences derived from SINEs in the mammalian genome. Genome Research, Vol. 16, No. 7, (July), pp. 864-874, ISSN 10889051

Okamoto, H. et al. (2010) Wnt2 expression and signaling is increased by different classes of antidepressant treatments. Biological Psychiatry, Vol. 68, No. 6, (September 15), pp. 521-527, ISSN 0006-3223

Okamoto, M. et al. (2011) Reduction in paracrine Wnt3 factors during aging causes impaired adult neurogenesis. The FASEB Journal, Vol. 25, No. 8, (August), pp. xx-xx, ISSN 0892-6638

Pagano S. F. et al. (2000) Isolation and characterization of neural stem cells from the adult human olfactory bulb. Stem Cells. Vo.18, No.4, pp. 295-300, ISSN 1066-5099

Palmer, T. D. et al. (1995) FGF-2-responsive neuronal progenitors reside in proliferative and quiescent regions of the adult rodent brain. Molecular and Cellular Neuroscience, Vol. 6, No. 5, (October), pp. 474-486, ISSN 1044-7431 
Paule, M. R. \& White, R. J. (2000) Survey and summary: transcription by RNA polymerases I and III. Nucleic Acids Research, Vol. 28, No. 6, (March 15), pp. 1283-1298, ISSN 03051048

Song, H. et al. (2002) Astroglia induce neurogenesis from adult neural stem cells. Nature, Vol. 417, No. 6884, (May 2), pp. 39-44, ISSN 0028-0836

Suh, H., et al. (2007) In vivo fate analysis reveals the multipotent and self-renewal capacities of Sox2+ neural stem cells in the adult hippocampus. Cell Stem Cell, Vol. 1, No. 5, (November), pp.515-528, ISSN 1934-5909

Tozuka, Y. et al. (2005) GABAergic excitation promotes neuronal differentiation in adult hippocampal progenitor cells. Neuron Vol. 47, No. 6, (September 15), pp. 803-815, ISSN 0896-6273

Ullu, E. \& Tschudi, C. (1984) Alu sequences are processed 7SL RNA genes. Nature, Vol. 312, No. 5990, November 8-14), pp. 171-172, ISSN 0028-0836 


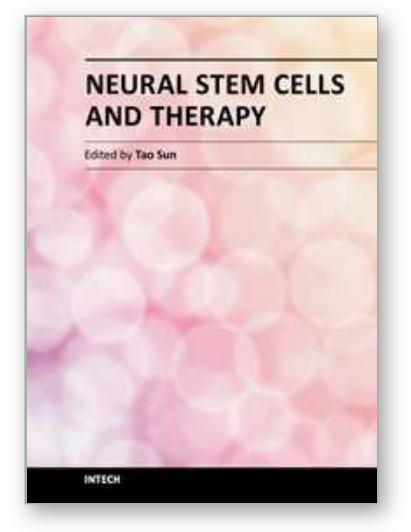

\author{
Neural Stem Cells and Therapy \\ Edited by Dr. Tao Sun
}

ISBN 978-953-307-958-5

Hard cover, 440 pages

Publisher InTech

Published online 15, February, 2012

Published in print edition February, 2012

This book is a collective work of international experts in the neural stem cell field. The book incorporates the characterization of embryonic and adult neural stem cells in both invertebrates and vertebrates. It highlights the history and the most advanced discoveries in neural stem cells, and summarizes the mechanisms of neural stem cell development. In particular, this book provides strategies and discusses the challenges of utilizing neural stem cells for therapy of neurological disorders and brain and spinal cord injuries. It is suitable for general readers, students, doctors and researchers who are interested in understanding the principles of and new discoveries in neural stem cells and therapy.

\title{
How to reference
}

In order to correctly reference this scholarly work, feel free to copy and paste the following:

Slawomir Antoszczyk, Kazuyuki Terashima, Masaki Warashina, Makoto Asashima and Tomoko Kuwabara (2012). Active Expression of Retroelements in Neurons Differentiated from Adult Hippocampal Neural Stem Cells, Neural Stem Cells and Therapy, Dr. Tao Sun (Ed.), ISBN: 978-953-307-958-5, InTech, Available from: http://www.intechopen.com/books/neural-stem-cells-and-therapy/active-expression-of-retroelements-inneurons-differentiated-from-adult-hippocampal-neural-stem-cell

\section{INTECH}

open science | open minds

\section{InTech Europe}

University Campus STeP Ri Slavka Krautzeka 83/A 51000 Rijeka, Croatia Phone: +385 (51) 770447 Fax: +385 (51) 686166 www.intechopen.com

\section{InTech China}

Unit 405, Office Block, Hotel Equatorial Shanghai No.65, Yan An Road (West), Shanghai, 200040, China 中国上海市延安西路65号上海国际贵都大饭店办公楼 405 单元 Phone: +86-21-62489820

Fax: +86-21-62489821 
(C) 2012 The Author(s). Licensee IntechOpen. This is an open access article distributed under the terms of the Creative Commons Attribution 3.0 License, which permits unrestricted use, distribution, and reproduction in any medium, provided the original work is properly cited. 